\title{
Development of Archives Management under the Background of E-commerce
}

\author{
Xiaohui Dai \\ Comprehensive Document Room, Taishan University, Tai'an, 271021, China
}

Key words: e-commerce, archives, management

\begin{abstract}
With its major medium as e-commerce, network allowed various countries in the world to form a unified market system by connecting the information networks in various countries in the world, which remarkably expanded the source of archives management. At the same time, this also created favorable conditions for the use of more scientific and more advanced approaches for archives management. Based on a brief introduction to e-commerce in this paper, the author analyzed main effects of e-commerce on archives management, and proposed several countermeasures for strengthening archives management under the background of e-commerce.

Considering the continuous development of information technology in the modern society, e-commerce has already entered many social fields as an indispensable part. At the same time, archives management under the background of e-commerce is also faced with the transformation of a new pattern, from traditional paper materials to information and networking management. Archives workers in various offices, enterprises and public institutions should positively adapt to this change, get well prepared in both thought and action, and make great efforts for archives management under the perspective of e-commerce with a brand new image.
\end{abstract}

\section{Introduction to E-commerce}

In the last century, the definition of e-commerce was discussed on the century e-commerce conference held in Paris (France). According to the discussion, e-commerce mainly refers to the implementation of e-management toward trade activities, so as to make it convenient for various related parties to apply the mode of electronic transaction in implementing commercial and market transactions in any form instead of face-to-face exchange or interview. As the integrity of many technologies, e-commerce mainly includes swap data, getting data and automatically captured data. Inevitably, e-commerce will develop into one of significant channels of communication for the implementation of business management and development in the modern information age. E-commerce is served as the product of modern science and technology. Its appearance and existence is closely correlated with information-based society and network world. Contemporary people live in the modern society. However, e-commerce is also just in such environment. Enterprises can apply international Internet to obtain more convenience and benefits than traditional commercial means of exchange. A large number of enterprises established e-commerce websites. It is faster and more convenient for customers to understand information through access to these websites when compared with dialing or consulting at the enterprises. Moreover, it is more beneficial for enterprises. The reason is that it is unnecessary to employees to be involved in specific transactions. As a result, plenty of costs are saved, and a lot of risks caused by improper exchange between customers and employees can be eliminated. At the same time, e-commerce also plays a positive role in adjusting operating management of enterprises. Certainly, one of outstanding features of e-commerce different from traditional commerce is that e-commerce can form brand new means of commerce. Enterprises can employ the network to broadcast advertisement or information can timely receive feedback information of customers after looking up relevant information, so as to introduce products of enterprises to the maximum extent, expend the market, obtain considerable information resources in need, and find out more customer resources with cooperative potential. Moreover, the application of various services provided by e-commerce can tremendously improve the production and management efficiency. 


\section{Main Effects of E-commerce on Archives Management}

(I) Effect on the archives management objective

With the appearance of e-commerce, demanders for archival information began to utilize network as the channel of archival information and two-way communication, so as to offer required contents of archival information to various providers. At the same time, this also makes it more and more difficult to determine the limit of archives subject. Considering the uncertainty of network commercial opportunities, it is possible to form e-commerce enterprises or disappear in an instant. As a result, it becomes more and more difficult to identify the subject of archives. Under the background of e-commerce, users of archival information always need more information about human resources and intellectual property. As a result, users can implement correct, rational and effective measures only upon a comprehensive understanding of relevant information, so as to avoid false decisions caused by insufficient prudence. Occurred social activities can be promptly filed by means of network. Moreover, such activities can be saved in relevant databases which can be queried by users. Archives users can absolutely utilize the network to enter the archives management system, so as to select and analyze necessary information in a more timely, effective and scientific way. At the same time, archives users can also effectively save transmission costs and space, control costs for product storage, and improve the degree of safety in commodity storage.

(II) Effect on development and utilization of archives management

Considering the remarkable integration of network environment toward the processing of modern information, almost all trading rights can enter different computer software modules. Thus, the degree of manual intervention can be reduced. Moreover, the security of archives and the authenticity of data can be guaranteed. In the information age and e-commerce age, indispensable duties under the original state of handwork need to be finished by many people. Under the environment of modern network, however, computer programs can be used to realize these duties. Archives managers in various enterprises must rationally utilize profound specialized knowledge and related economic knowledge to properly analyze and evaluate archival information management, and assist relevant leaders in making correct decisions. For better adaption to various requirements in the e-commerce age, modern network archives management software must be provided with the following new features: i) realize modern management means of archives in terms of management mode, such as cooperative processing, remote processing, online transmission management and centralized management of archives, and support new ways in the information age, such as online working and mobile working; ii) provide relevant functions (such as remote inquiry) on the basis of the present archives management software in terms of software function; and iii) software must take archives management as the core element. Main techniques of the network electronic commerce system should be used for development and operation. Moreover, archives management software should also be easy for operation and maintenance, so as to allow archives management workers to use this software with high proficiency.

(III) Effect on the carrier format of archives

Considering wide promotion and application of computer networking technology and the large-scale appearance of new file formats (e.g. E-mail and electronic contract), especially in e-commerce activities of organs, enterprises and public institutions, organs, enterprises and public institutions are certain to have more and more electronic documents to be saved in USB flash disk, compact disk and computer hard disk in daily management. Above documents are used to record various activities related to workers in relevant enterprises. Compared with traditional paper documents, however, the difference is that these documents have distinct characteristics, such as non-manual reading, storage high density, detachability between information and carrier, integration of various carrier information, and high dependency on system. For organs, enterprises and public institutions, how to save and utilize more and more electronic documents has already become an important issue requiring urgent settlement for the development of archival work under the new situation.

(IV) Effect on the mode of archives management

Electronic information technology got large-scale application under e-commerce activities. As a 
result, the human society was promoted to change its management mode. More importantly, a large number of cultural concepts also got tremendous changes. Under the context of e-commerce, all contents cannot be known by sight. Relevant features of electronic document become more and more complicated. Thus, it is necessary for archival workers to have a perfect mastery of confirming the truth, reliability and integrity of electronic documents. For more timely and accurate obtainment of necessary documents and archival information under the information age, all institutional frameworks are required to utilize computer and network technique to meet the needs for information. Archives workers must perfect themselves in or even be proficient in relevant computer technologies, so as to use the automated management mode to provide relevant archival information to related leaders and workers in a faster and more accurate way, and to serve scientific decision-making.

(V) Effect on the flow of archives management

New changes on archives carriers in organs, enterprises and public institutions also resulted in new changes on the flow of archives management. Specially, new changes occurred in time, content and sequence of electronic document management. Thus, enterprises are required to make great efforts to realize front control and whole-course management of archives under the background of e-commerce. Firstly, new changes took place in the time of archives management. Electronic document has such deficiencies as easy change and delete. Such deficiencies decide the fact that the archives management time under the background of e-commerce must be advanced to the formation period of electronic document. Only in this way, electronic documents can be prevented from any issue caused by damage or manual change after the generation of such documents. Secondly, new changes took place in the content of archives management. All electronic documents were generated under the electronic environment. Therefore, these documents highly depend on computer software and hardware. Thus, it requires electronic documents to be saved together with matched generation software or management systems upon the formation of these electronic documents. For document identification, identifiers are required to judge technical state of documents in the identification process of electronic documents. All above items cannot be designed in traditional archives identification. Thirdly, new changes took place in the sequence of archives management. Considering that filing, arrangement, identification, generation of metadata and other work processes of archives management need to be proceeded in the generation phase of electronic documents, the flow of electronic document management should be run through the whole generation process of electronic documents. This is completely different from traditional paper archives management procedure.

\section{Several Countermeasures for Strengthening Archives Management under the Background of E-commerce}

(I) Form the consciousness and sense of responsibility of nationwide archives management

Maintain integrity and accuracy of archives: it needs to be guaranteed by the operation flow instead of obtaining by arrangement at last. At present, Chinese organs, enterprises and public institutions are forming document texts in different types. Moreover, most texts are saved as electronic documents, and continuously used in the electronic form. However, various circumstances (such as not timely, proper or accurate enough collection of archives) often appear in the management process of electronic documents. This is directly correlated with relatively weak archives management consciousness of workers in organs, enterprises and public institutions. Nowadays, the management becomes more and more stringent in various organs, enterprises and public institutions. Moreover, it is inevitably necessary for various examinations and assessments to inspect and verify archival data under the background of e-commerce. Therefore, organs, enterprises and public institutions must pay high attention to and form the whole archives management consciousness, so as to effectively promote the improvement to the management level.

(II) Enhance the filing work of archives under the background of e-commerce

With the vigorous promotion of automatic archives works in organs, enterprises and public institutions and the successive appearance of various computer aided technologies, many enterprises 
are exploring for an approach to replace paper documents with electronic ones. Therefore, we should arrange and file electronic documents that can reflect economic activities and commercial activities of organs, enterprises and public institutions and that have relevant examination value and use value while selecting filing documents. Certainly, electronic documents must be truly and effectively filed. Filed texts of electronic texts must be of finalized texts. If graphic documents have already been changed, these documents must be saved in versions consistent with the product technical state at that time. Besides, we should also decide whether to file drafts of various kinds of electronic documents or not according to specific needs. At the same time, electronic documents can be classified, combined and retrieved in computer by using multiple angles, so as to perfectly present the relations of these documents with multiple aspects, such as archives source, archives content, formation time and pattern. Therefore, it is unnecessary to be classified or rolled as paper archives. In the saving process of electronic documents, relevant archives keepers can convert electronic documents into paper ones, because it is faster and more convenient to arrange archives under the background of e-commerce than paper archives. Considering that there is no legal basis or technical condition in adequate support to the saving of electronic documents at present, it is unable to replace paper documents by electronic documents.

(III) Promote working conditions of archive keeping under the background of e-commerce

Archives carrier under the background of e-commerce is completely different from the saving of paper archives in the traditional sense. Therefore, there are also lots of differences in terms of saving requirement. To be specific, the storage medium used for the formation of electronic archives under the background of e-commerce is high-capacity carrier, for instance, USB flash disk and light disk. At the same time, archival repository in the e-commerce age has new and higher requirements for dust prevention, prevention against harmful gases and other conditions. The archival repository should be clean, dust free, in good ventilation and able to present ultraviolet light exposure or magnetic field interference. Moreover, reasonable humidity and temperature should be maintained. Besides, archives management staff should also regularly inspect whether light disk and other storage media are damaged or infected with virus. In case of any issue, backup files should be used immediately for copying, so as to present any loss of archival data.

(IV) Promote archives utilization under the background of e-commerce

The appearance of archives under the background of e-commerce really changed traditional archives reading, borrowing and other utilization modes, letting the utilization of electronic records free from the restraint from time and space. The contents of archives under the background of e-commerce can be completely provided by automatic retrieval via computer. Therefore, archives users can utilize archives by computer under the background of e-commerce, so as to remarkably reduce the retrieval and transferring pressure of archives workers.

\section{Conclusion}

To sum up, archival work should continuously adapt to modern management needs under the new situation, actual development conditions of organs, enterprises and public institutions, and changes in the content of e-commerce archives, so as to make continuous improvement to the archives management level of this organization and the quality of relevant workers and to make better response to challenges in the information age.

\section{References}

[1] Chen Zhonghai, Science of Archives Management [M], Zhengzhou: Henan People’s Publishing House, 2006;

[2] Shen Jiangsheng, Two Questions about E-commerce Archives Management [M], Heilongjiang Shizhi, 2012 (12);

[3] Yang Jihui, Analysis on Intelligence Trend of E-commerce Archives Management [J], Office 
Operations, 2013 (9);

[4] Wang Zhuo, Analysis on Intelligence of E-commerce Archives Management [J], Heilongjiang Shizhi, 2014 (20) 